\title{
Non-Newtonian Open Channel Flow — A Simple Method of Estimation of Laminar/Turbulent Transition and Flow Resistance
}

\author{
T.G. Fitton Australian Tailings Consultants, Australia
}

\begin{abstract}
A simple method is presented for the calculation of the Reynolds number at which a non-Newtonian fluid reaches laminar/turbulent transition in an open channel. As well, a simple approach to the estimation of head losses is presented for non-Newtonian open channel flows in the laminar, transitional or turbulent regimes, enabling the flow depth and velocity to be predicted for various fluids, flow rates and channel geometries. Validation of this approach is provided by a diverse range of 776 experimentally measured open channel flow depths, with good agreement found between the predicted and observed values.
\end{abstract}

\section{Introduction}

The prediction of the point of transition from laminar to turbulent flow for non-Newtonian fluids flowing in open channels is not yet easily or accurately achieved (Haldenwang, 2003). Methods have been presented for predicting the laminar/turbulent transition point that require pipe loop experiments to be undertaken (Wilson, 1988), while other workers have presented empirical equations that are relatively difficult to apply (Haldenwang et al., 2002; Haldenwang, 2003). There are a few equations available for predicting flow resistance for water in open channels, such as the Manning, Chezy and Darcy-Weisbach equations, but none of these provides a reliable means for predicting friction losses for non-Newtonian fluids flowing in open channels (Chadwick et al., 2004).

A crude method is presented here for the prediction of the laminar/turbulent transition for a given nonNewtonian open channel flow scenario, and the subsequent prediction of the friction loss for that same scenario. This method is presented as a practical basis for the design of flumes and launders, and the simple analysis of other open channel flow situations such as river flows, sewage and sludge transport and food processing.

\section{Open channel flow resistance}

The Darcy-Weisbach equation was developed for determining the head loss in pipe flow situations, but can be readily adapted to open channel calculations (and often is). Unlike the Manning and Chezy equations, the Darcy-Weisbach equation is theoretically derived from first principles. It applies to both turbulent and laminar flow conditions. The common form of the Darcy-Weisbach equation is as follows (Chadwick et al., 2004):

$$
h_{f}=f_{D} \cdot \frac{L}{D} \cdot \frac{V^{2}}{2 g}
$$

where $h_{f}$ is the head loss in a pipe of length $L$ and diameter $D . V$ is the average velocity of flow, $g$ is the acceleration due to gravity and $f_{D}$ is defined as the Darcy friction factor. It is noted that this friction factor is not to be confused with the Fanning friction factor, $f_{N}$, which is equal to $f_{D} / 4$. This is the pipe flow form of the equation, but it can be adapted to open channel flow using the following geometric relationship (Chadwick et al., 2004):

$$
D=4 R_{H}
$$


where $R_{H}$ is the hydraulic radius of the channel, equal to the cross sectional area of flow divided by the wetted perimeter of the channel. Another equation that further simplifies the application of the DarcyWeisbach equation to open channel flow is the geometric relationship (Chadwick et al., 2004):

$$
S_{0}=\frac{h_{f}}{L}
$$

where $S_{0}$ is the slope of the channel bed for a fluid in uniform flow. Equations 1, 2 and 3 can be combined to yield the following open channel form of the Darcy-Weisbach equation:

$$
f_{D}=\frac{8 g R_{H} S_{0}}{V^{2}}
$$

The Darcy-Weisbach equation provides one main advantage over the Manning and Chezy equations; it can be readily applied to non-Newtonian fluids, with the viscous behaviour of the fluid being contained solely within the Reynolds number term.

In terms of quantifying the friction aspects of channel bed, the same problem comes with applying the Darcy-Weisbach equation; is it essential to determine an appropriate value for the Darcy friction factor, $f_{D}$. Significant experimental research has been done in an effort to predict the value of $f_{D}$ for various scenarios. The most relevant of all of the various research studies was that done by Nikuradse (as quoted by Colebrook and White,1937) in the early 1930s, in which he conducted pipe flow experiments with the inside surface of the pipe coated with sand grains of a uniform diameter, $k_{s}$. He discovered that the pressure loss in a pipe was not affected by the roughness of the pipe at relatively low pressure gradients, despite the flow being fully turbulent. Conversely, he also discovered that the viscosity of the fluid had no effect when the pressure gradient was very high, but in between these two extremes, he found that the viscosity and the roughness both had an effect on the friction losses in the pipe. In the mid 1930s, Colebrook and White further investigated the findings of Nikuradse. In 1937 they presented a paper in which they combined the two correlations observed by Nikuradse, thereby introducing the Colebrook-White equation for enabling the determination of an $f_{D}$ value for a given turbulent flow of a Newtonian fluid in a pipe (Colebrook and White, 1937). Colebrook and White then presented some typical $k_{s}$ values for commonly used pipe materials. Various other workers have since published $k_{s}$ values for other surfaces (Abulnaga, 2002).

The common pipe flow form of the Colebrook-White equation is shown below (Chadwick et al., 2004).

$$
\frac{1}{\sqrt{f_{D}}}=-2 \log _{10}\left[\frac{k_{s}}{3.7 D}+\frac{2.51}{\operatorname{Re} \sqrt{f_{D}}}\right]
$$

It can be seen that this equation is implicit of $f_{D}$, and so is not simple to apply. It can be used iteratively to determine an $f_{D}$ value that will be accurate to a few decimal places after 4 or 5 iterations, but to do this manually is a tedious process. For this reason, the Colebrook-White equation has been used to generate the transition and turbulent regions of the popular Moody diagram, which enables the determination of a value for $f_{D}$ for a pipe if the pipe roughness, $k_{s}$, and the Reynolds number, Re, are known. For pipe flow calculations the Moody diagram is very popular and convenient.

The Colebrook-White equation can be applied to open channel flow by replacing $D$ with $4 R_{H}$ (Equation 2):

$$
\frac{1}{\sqrt{f_{D}}}=-2 \log _{10}\left[\frac{k_{s}}{14.8 R_{H}}+\frac{2.51}{\operatorname{Re} \sqrt{f_{D}}}\right]
$$

This version of the equation has also been used to generate Moody diagrams that are applicable to open channel flow (Yen, 2002). 
For Newtonian fluids in open channel flow, the Reynolds number is calculated by Haldenwang (2003):

$$
\operatorname{Re}=\frac{4 \rho V R_{H}}{\mu}
$$

For non-Newtonian fluids in open channel flow, Haldenwang et al. (2002) presented the following equation for calculating the Reynolds number, with the rheological properties of the fluid expressed in terms of the three Herschel-Bulkley fitting parameters $\tau_{y}, K$ and $n$ :

$$
\operatorname{Re}_{H B}=\frac{8 \rho V^{2}}{\tau_{y}+K\left[\frac{2 V}{R_{H}}\right]^{n}}
$$

In laminar flows the Darcy friction factor is calculated with the following equation, which has been empirically found to describe laminar flows in narrow open channels (Chadwick et al., 2004):

$$
f_{D}=\frac{64}{\operatorname{Re}}
$$

This equation holds true for both Newtonian and non-Newtonian fluids.

\section{Prediction of the state of flow}

This approach categorises open channel flows into two states; either laminar or turbulent (effectively ignoring transitional flow behaviour because of the ability of the Colebrook-White equation to describe both turbulent and transitional flows reasonably well). The prediction of which of these two states applies for a given open channel flow scenario is very simple: the Darcy friction factor is calculated with both the HagenPoiseuille equation and the Colebrook-White equation. If the Darcy friction factor calculated with the Hagen-Poiseuille equation is larger than that calculated with the Colebrook-White equation, the flow is laminar; otherwise it is turbulent. Because of the implicit nature of the Colebrook-White equation, it is recommended that a spreadsheet program is used to perform the necessary iterative calculations efficiently. The input parameters required to predict the state of flow are as follows:

- The channel geometry, such that the cross sectional area of flow and the wetted perimeter can be calculated as a function of the depth.

- The depth of flow (m).

- $k_{s} \quad$ the channel roughness $(\mathrm{m})$.

- $Q \quad$ Flow rate $\left(\mathrm{m}^{3} / \mathrm{s}\right)$.

- $S_{0} \quad$ Channel bed slope, assuming uniform flow conditions (expressed as a fraction).

- $\rho$ Density of the fluid $\left(\mathrm{kg} / \mathrm{m}^{3}\right)$.

- $\mu \quad$ Viscosity of the fluid (Pa.s) for Newtonian situations.

For a non-Newtonian fluid the Herschel-Bulkley model parameters are required:

- $\tau_{\mathrm{y}} \quad$ Yield stress of the fluid (Pa).

- $K \quad$ Fluid consistency index $\left(\mathrm{Pa} . \mathrm{s}^{n}\right)$.

- $n \quad$ Fluid behaviour index. 
These Herschel-Bulkley parameters can be accurately derived from rheometry experiments, which require approximately $200 \mathrm{ml}$ of fluid as a sample.

\section{Prediction of the point of laminar/turbulent transition}

From the same spreadsheet used to predict the state of flow, the point of laminar/turbulent transition can be calculated by changing the depth of flow until the Hagen-Poiseuille equation calculates the same Darcy friction factor as the Colebrook-White equation. The "solver" or "goal seek" functions of the spreadsheet program will make this iterative process virtually instant. Once the two equations are calculating the same $f_{D}$ value, the transition point can be expressed in terms of the corresponding Reynolds number value.

\section{Prediction of depth of flow}

For a given situation in which the channel geometry, roughness, slope, flow rate, fluid rheology and density are known, the depth of flow in the channel can be predicted using the following steps:

1. Guess an initial value for the depth.

2. Calculate the cross sectional area of flow, $A$, and the wetted perimeter, $P$, as a function of the guessed depth of flow.

3. Calculate $R_{H}$, the ratio of $A / P$.

4. Calculate $V$, the average velocity in the channel (equal to $Q / A$ ).

5. Calculate the Reynolds number using equation 8 .

6. Calculate the Darcy friction factor, $f_{D}$, using equation 6.

7. Calculate the Darcy friction factor, $f_{D}$, using equation 9.

8. Use the greater of the two $f_{D}$ values calculated in steps 6 and 7 to predict the slope of flow (even though the slope is already known) using the Darcy-Weisbach equation.

9. Change the initial depth value, then repeat steps 2 to 8 until the slope calculated in step 8 is equal to the actual slope of the channel. At this point the depth of flow is predicted.

This sequence of steps will enable the depth of flow to be predicted. A similar approach could be used to predict the head loss or some other parameter such as the slope of the channel or the flow rate, providing the remaining parameters listed above in Section 3 are known.

\section{Validation of the prediction method}

Four independently collected sets of data have been run through the depth prediction method presented above in Section 5:

1. The first data set, containing 49 points, was collected by the author with a $10 \mathrm{~m}$ tilting flume with two semi-circular cross sections of diameters 340 and $415 \mathrm{~mm}$, testing thickened tailings slurries at two separate mine sites, with concentrations ranging between 25 and $68 \%$ by weight. The inclination of the flume was varied from 0.0075 to 0.06 , and the rheological characteristics of the slurries were measured with a cup and bob rheometer. (Fitton et al., 2006) This data set has been referred to as "Field flume data".

2. The second data set, containing 623 data points, was collected by Rainer Haldenwang (Haldenwang and Slatter, 2006). He used three tilting flumes of rectangular cross section, with respective widths of 75,150 and $300 \mathrm{~mm}$. With slopes varying from 1 to 5 degrees, he tested four different non-Newtonian fluids in his flumes:

- Kaolin slurry of various volumetric concentrations, ranging from 3 to $10 \%$ (240 points).

- Bentonite slurry of 3 and $6 \%$ concentration by volume (117 points).

- Carboxymethylcellulose (CMC) solution of various volumetric concentrations, ranging from 1 to $3.8 \%$ (232 points).

- Carbopol solution of $1 \%$ concentration by volume (34 points). 
Haldenwang's apparatus was fitted with an in-line tube viscometer for measuring the rheological parameters of each fluid at the same time that the fluid was circulated through his flume. This data set has been referred to as "Haldenwang data".

3. The third data set, containing only 9 data points, was published by Seckin et al. (2006). This data was collected in a $22 \mathrm{~m}$ long tilted rectangular flume with a width of $398 \mathrm{~mm}$. All nine runs were done with water, with the flume set to a fixed slope of $2.024 \times 10^{-3}$. This data set has been referred to as "Seckin data".

4. The fourth data set, containing 95 data points, was published by Fitton (2007). This data was collected using a $5.4 \mathrm{~m}$ long glass flume of circular cross section, with an internal diameter of $50 \mathrm{~mm}$. Fluids tested in this flume included carbopol solution of two different concentrations, carboxymethylcellulose (CMC) solution of 11 different concentrations and water. Flume slopes varied between 0.2 and $7.7 \%$. This data set has been referred to as "small flume data".

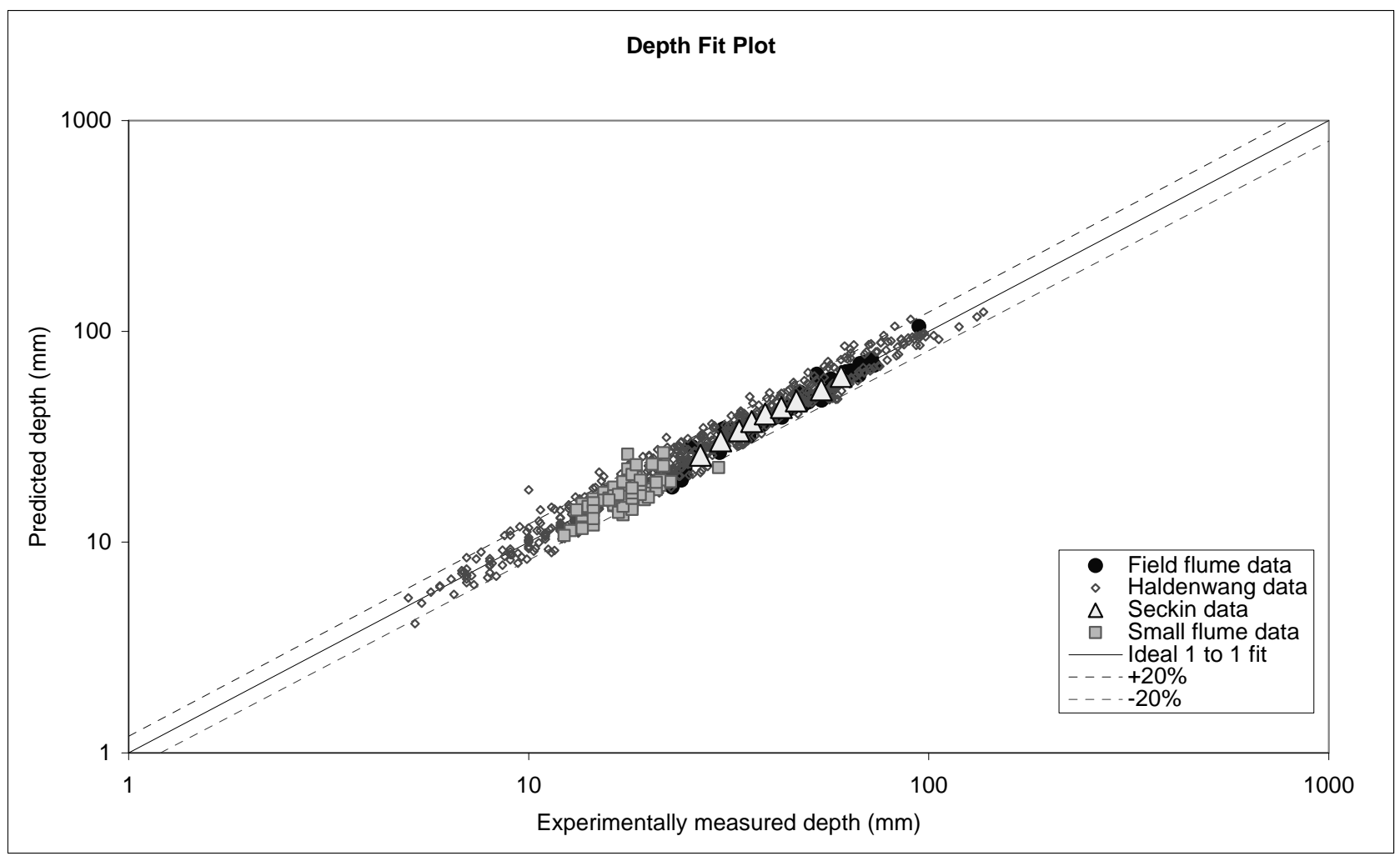

Figure 1 Fit plot featuring all 776 points from the 4 data sets

A fit plot featuring the total number of points from these four data sets is presented in Figure 1. The accuracy of the flow state prediction can be effectively indicated by considering the 623 data points of Haldenwang's data that were processed. Three-hundred-and-seventy-eight of these were found to be flowing in the laminar regime, with the corresponding Reynolds numbers for these points ranging from 0.06 to 1002 . The remaining 245 points from Haldenwang's data set were found to be in the transition and turbulent regimes, with corresponding Reynolds numbers ranging from 996 to 124000 . The maximum laminar Reynolds number of 1002 and the minimum transition Reynolds number of 996 are both reasonable values in relation to those reported in the literature (Lowe, 2003).

To illustrate the workings of this analysis, a Moody diagram has been presented as Figure 2 that features some actual experimental data plotted alongside corresponding predicted points. On this graph the data from Haldenwang's open channel experiments with a $10 \%$ concentrated mixture of Kaolin clay in water are plotted, as are the points from Haldenwang's experiments with $2.8 \% \mathrm{CMC}$ solution. In addition to these plotted points, the laminar line (generated by Equation 9) and the transition/turbulent lines (generated with Equation 6) are shown. It is these lines that have been assumed to approximate the fluid behaviour in the 
application of this simple method of predicting open channel flow resistance, but as can be seen for the CMC points in the Reynolds number range of 2000 to 4000, this approximation is less comparable to the actual values recorded by Haldenwang. On this basis it can be stated that this simple method of flow analysis is less accurate for transitional flow regimes than it is for laminar or fully turbulent regimes.

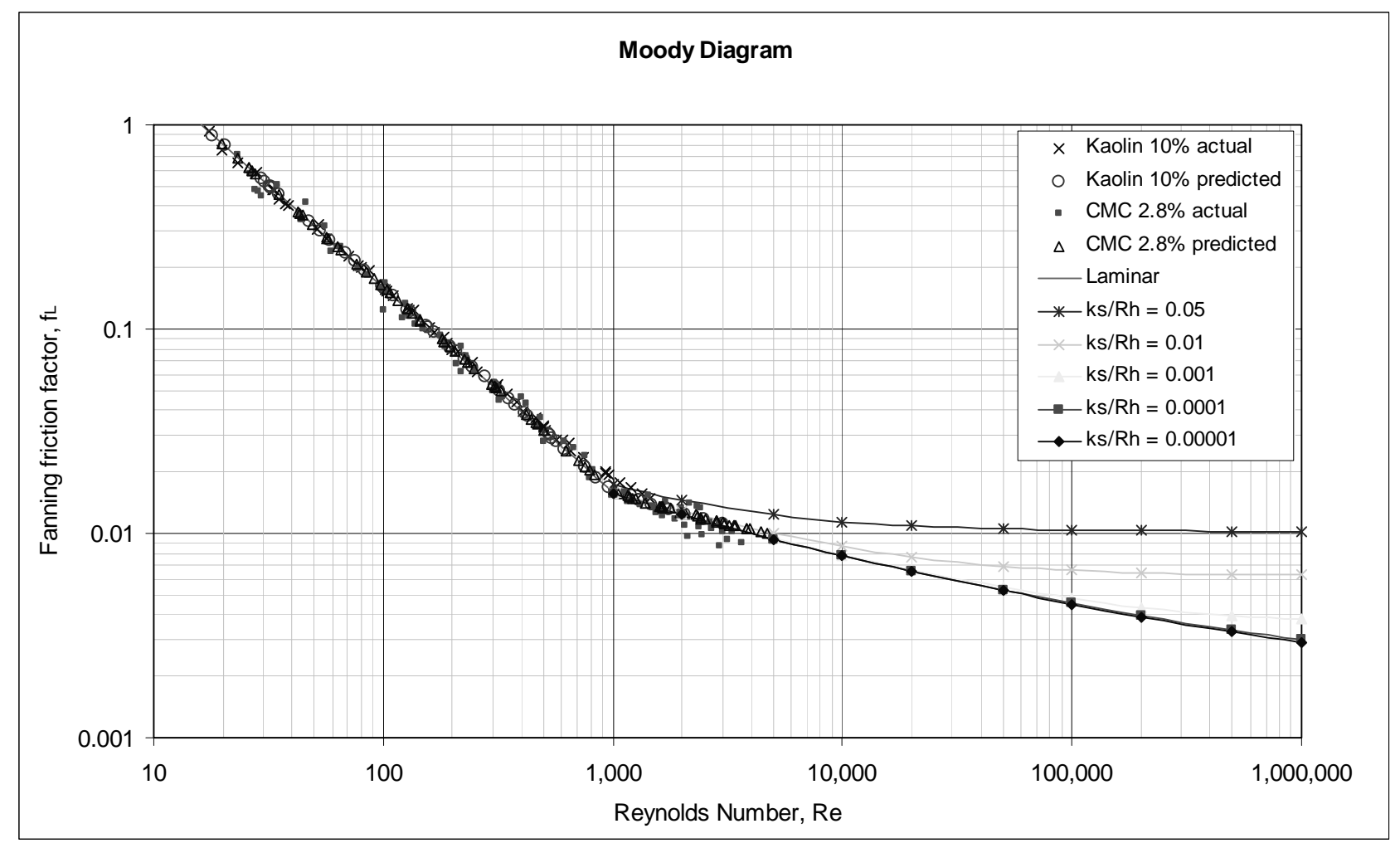

Figure 2 Moody diagram showing open channel experimental data from Haldenwang's $10 \%$ Kaolin mixture and $2.8 \%$ CMC solution tests, alongside predicted points

\section{Conclusion}

A simple method has been presented for predicting the depth of uniform flow of both Newtonian and nonNewtonian fluids in an open channel. This approach also enables the state of flow to be predicted (i.e. whether the flow is laminar or turbulent), as well enabling the point of laminar/turbulent transition to be predicted. With the use of a spreadsheet program, the depth (or some other open channel flow parameter) can be predicted quickly. Strong validation has been provided to demonstrate the accuracy of depth predictions made with this method, with good agreement from four independently collected experimental data sets featuring a range of fluids.

\section{Acknowledgements}

A special mention goes to Andrew Chryss at RMIT for guidance and assistance with the small flume and field flume experimental work. Thanks also goes to Dr Rainer Haldenwang from the Cape Peninsula University of Technology, who provided the author with access to his considerable experimental data set. Acknowledgement is also given to Peter Lam, Australian Tailings Consultants (ATC), Behnam Pirouz (NICICO) and Alex Walker, ATC for their input and assistance in the field flume experimental programmes. Finally, recognition goes to those who have provided support at Australian Tailings Consultants, Sunrise Dam Gold mine and Peak Gold mine. 


\section{References}

Abulnaga, B. (2002) Slurry Systems Handbook, McGraw-Hill, New York.

Chadwick, A., Morfett, J. and Borthwick, M. (2004) Hydraulics in civil and environmental engineering, Fourth edition, Spon press, London and New York.

Colebrook, C.F. and White, C.M. (1937) Experiments with Fluid Motion in Roughened Pipes, Proc. R. Soc. London, Ser. A.

Fitton, T.G., Chryss, A.G. and Bhattacharya, S.N. (2006) Tailings beach slope prediction: A new rheological method, Int. J. Surface Mining, Reclamation and Environment, Vol. 20, No. 3, pp. 181-202.

Fitton, T.G. (2007) Tailings beach slope prediction, PhD thesis, RMIT University, Melbourne, Australia.

Haldenwang, R. (2003) Flow of non-Newtonian fluids in open channels. Unpublished D.Tech thesis. Cape Technikon, Cape Town.

Haldenwang, R.H. and Slatter, P.T. (2006) Experimental procedure and database for non-Newtonian open channel flow, Journal of Hydraulic Research, Vol. 44, No. 2, pp. 283-287.

Haldenwang, R., Slatter, P.T. and Chhabra, R.P. (2002) Laminar and Transitional flow in Open Channels for nonNewtonian Fluids. 15th International Conference on Hydrotransport. Banff, Canada, pp. 755-768.

Lowe, S. (2003) Omission of Critical Reynolds Number for Open Channel Flows in Many Textbooks. J. Prof. Issues in Engrg. Educ. and Pract. 129(1), pp. 58-59.

Seckin, G., Seckin, N. and Yurtal, R. (2006) Boundary shear stress analysis in smooth rectangular channels, Canadian Journal of Civil Engineering; Mar 2006, 33, 3, pp. 336-342.

Wilson, K.C. (1988) Slurry transport in flumes. Slurry handling design of solid-liquid systems. N.P. Brown and N. I. Heywood, Elsevier Applied Science, pp. 167-180.

Yen, B.C. (2002) Open channel flow resistance, Journal of Hydraulic Engineering, Vol. 128, No. 1, pp. 20-39. 